\title{
Myxoid liposarcoma associated with adalimumab treatment: A case report
}

\author{
CARMEN RODRÍGUEZ-CERDEIRA ${ }^{1,2}$, JOSÉ IGNACIO NEISSA-VASQUEZ ${ }^{3}$, MIGUEL CARNERO-GREGORIO ${ }^{1}$, \\ CONCEPCIÓN FIAÑO-VALVERDE ${ }^{3}$ and VICTOR MUÑOZ-GARZÓN ${ }^{4}$ \\ ${ }^{1}$ Efficiency, Quality and Costs in Health Services Research Group (EFISALUD), Health Research Institute, \\ SERGAS-UVIGO; ${ }^{2}$ Department of Dermatology, Meixoeiro Hospital, CHUVI, 36200 Vigo; \\ ${ }^{3}$ Department of Histopathology, Álvaro Cunqueiro Hospital, CHUVI, 36312 Vigo; \\ ${ }^{4}$ Department of Radiotherapy, Meixoeiro Hospital, CHUVI, 36200 Vigo, Spain
}

Received December 20, 2018; Accepted February 26, 2019

DOI: $10.3892 / \mathrm{mco} .2019 .1817$

\begin{abstract}
Biological agents that suppress inflammation, such as tumour necrosis factor (TNF- $\alpha$ ) inhibitors, are being successfully used at an increasing frequency for the treatment of chronic inflammatory diseases, such as psoriasis. However, these drugs are not free of side effects, and although the general rates of malignancy in patients using anti-TNF- $\alpha$ therapies are not high, certain tumours of cutaneous origin, particularly carcinomas, have been reported. We herein present the case of a 47-year-old female patient with moderate-to-severe psoriasis for 20 years under treatment with adalimumab administered at the standard dose of $40 \mathrm{mg}$, injected subcutaneously each fortnight, with good efficacy. To the best of our knowledge, this is the first reported case of a low-grade (grade 1) myxoid liposarcoma in a patient receiving treatment with adalimumab since 2009. A review of the relevant literature was also conducted. Continuous investigation of such cases is crucial in order to elucidate the iatrogenic risk of rare cancers, such as myxoid liposarcoma, in patients undergoing treatment with currently available as well as future biological therapies.
\end{abstract}

\section{Introduction}

Psoriasis is a chronic immune-mediated disease characterized by thick red cutaneous lesions that are covered by gray or silvery white patches that tend to peel. Plaque psoriasis is the most common form, affecting $~ 90 \%$ of patients with psoriasis. Psoriatic lesions typically present as raised areas of erythematous skin covered with white scales. The lesions can appear

Correspondence to: Dr Carmen Rodríguez-Cerdeira, Department of Dermatology, Meixoeiro Hospital, CHUVI, Meixoeiro Street, w/n, 36200 Vigo, Spain

E-mail: carmencerderia33@gmail.com

Key words: adalimumab, moderate-to-severe psoriasis, myxoid liposarcoma, adverse events throughout the body, although the scalp, elbows, lower back, navel and knees are the most common sites of involvement (1).

The introduction of biological agents has revolutionized the management of moderate-to-severe psoriasis, and these drugs are considered as second-line therapy following administration of first-line systemic medications to control the disease. Since the introduction of the first drug belonging to this family [an anti-tumour necrosis factor- $\alpha(\mathrm{TNF}-\alpha)$ indicated for psoriasis in adults], several other agents have become available (2).

Adalimumab is one of the most extensively investigated biological agents. This drug is indicated for the treatment of adults with moderate-to-severe disease manifesting as plaques that are refractory to treatment, or is used in patients with contraindications or intolerance to other systemic therapies, including cyclosporin, methotrexate or psoralen and ultraviolet A therapy (3).

Adalimumab is administered as a biweekly subcutaneous injection. Its use is associated with a high risk of infection, particularly tuberculosis (as is observed with the use of other TNF- $\alpha$ inhibitors) (3). The evaluation of the risk of malignancy in patients with psoriasis receiving treatment with adalimumab and other TNF- $\alpha$ inhibitors may be challenging due to the following reasons: i) Possible dysregulation of the immune system is known to occur in these patients; ii) the patients may receive other immunomodulatory drugs; iii) the duration of exposure to these drugs varies between patients; and iv) only few reports in the literature have described TNF- $\alpha$ inhibitor-induced tumours (4).

\section{Case report}

A 47-year-old woman was diagnosed with psoriasis 20 years prior to presentation. The patient was treated with methotrexate, phototherapy and other topical treatments, and had been receiving adalimumab since 2009. Following remission, psoriasis has been completely controlled from 2016 onwards. In the beginning of 2017, a lesion compatible with an intramuscular myxoma was detected in the patient's right gluteus maximus on magnetic resonance imaging examination (Fig. 1). At that time, adalimumab administration was discontinued. Subsequently, an echocardiography-guided 


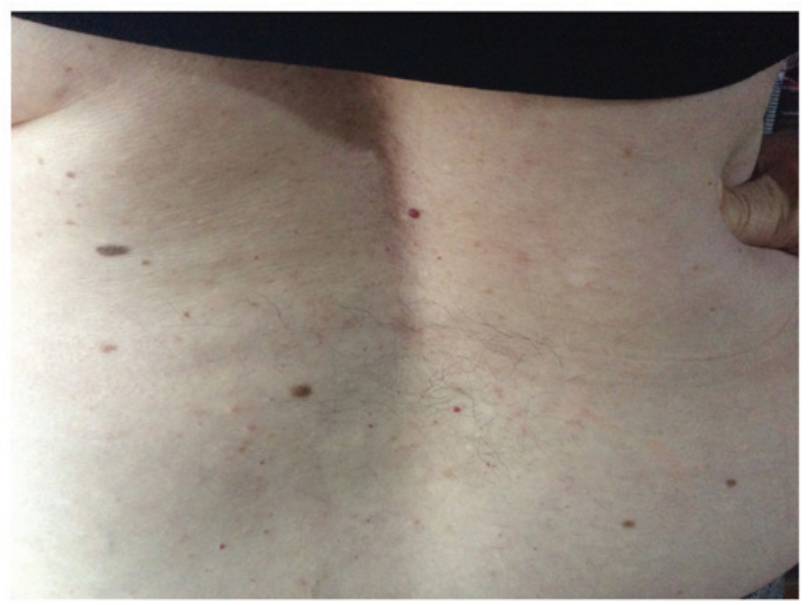

Figure 1. Magnetic resonance imaging shows a lesion in the right gluteal region.

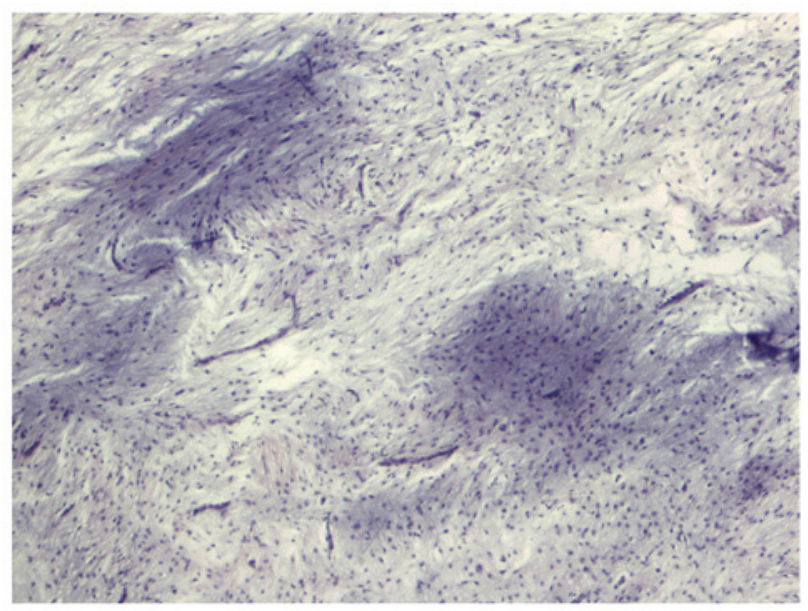

Figure 2. Histopathological examination revealed that the mass consisted of a typically myxoid stroma with small-sized spindle-shaped cells. A delicate network of thin-walled, elongated branched capillaries with the tendency to form a focal plexiform pattern is observed. Necrosis is not apparent, mitoses are practically absent, and nuclear pleomorphism is not clearly visible. Ki67 positive (haematoxylin and eosin staining; magnification, $\mathrm{x} 4$ ).

percutaneous biopsy was performed, and a low-grade myxoid tumour was initially diagnosed. In May 2017, surgery was performed under spinal anaesthesia, with wide resection of the tumour. The resected tissue specimen measured 7x7x9 cm; a longitudinal section revealed a whitish, shiny and gelatinous pseudotrabeculated tumour sized $4 \times 3.5 \times 3.7 \mathrm{~cm}$. The tumour was well-delineated and encapsulated with a tendency for spontaneous enucleation. The tumour was surrounded by muscle and adipose tissue. The patient was diagnosed with low-grade (grade 1) myxoid liposarcoma. Histopathological examination of the tumour revealed a myxoid mesenchymal neoplasm with variable cell density between mild and moderate and fusiform cells showing a non-specific distribution pattern (Figs. 2 and 3). Immunohistochemistry demonstrated that the Ki67 proliferative index was $1 \%$, and p53 expression was not observed. The patient received radiotherapy between July and September 2017. After time activity curve simulation, threedimensional planning was performed to administer a 60-Gy

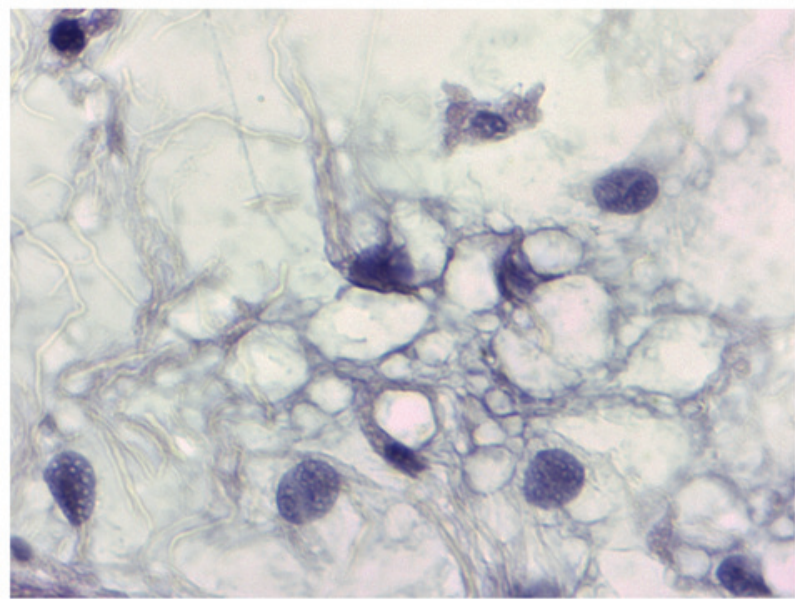

Figure 3. Histopathological image showing two univacuolate lipoblast-type cells with peripheral displacement of the nucleus (haematoxylin and eosin staining; magnification, x60).

dose into the surgical bed with wide margins, in 2 phases. During the first phase, an additional 46 Gy was administered into the surgical bed with wide margins. During the second phase, an additional $14 \mathrm{Mv}$ was administered into the surgical bed with the lower margins. The patient tolerated the treatment well with no significant toxicity. Regarding the follow-up, the patient is still periodically monitored by the Dermatology and Radiotherapy Service and there has been no recurrence data in the last monitored follow-up.

\section{Discussion}

Psoriasis is an autoimmune chronic inflammatory disease of the skin that manifests as oedematous and inflamed cutaneous lesions, with a variable clinical presentation and aetiology (5), which is associated with a high risk of skin cancer development (6).

Treatment for psoriasis ranges from topical preparations, such as corticosteroid-based ointments, to systemic therapy, including methotrexate, retinoids, cyclosporin A or phototherapy, which may be used as combination therapy or monotherapy. However, these aforementioned therapeutic options have limited efficacy and are associated with adverse effects (7). Thus, in recent years, the use of biological agents, such as TNF- $\alpha$ inhibitors (e.g., adalimumab) is becoming increasingly popular.

Recently, a class of drugs referred to as biological agents (or biologics) has been developed to treat several diseases, including cancer, multiple sclerosis, diabetes, rheumatoid arthritis, and/or psoriasis (6). The active ingredient in these biological agents is usually a protein (e.g., anti-TNF- $\alpha$ ) that exerts a therapeutic effect. Thus, these agents mimic the actions of proteins and biological processes in the host, suppress the symptoms of the disease and prevent or delay its evolution. However, the use of these immunomodulatory agents is associated with a high risk of serious infections and neoplasms.

Despite evidence-based studies supporting its efficacy, adalimumab is known to be associated with the development of skin tumours in a proportion of the patients. 
A previously published report described a case of adalimumab-induced epithelioid myxofibrosarcoma in a patient with psoriatic synovitis. The 65 -year-old patient described in that report was treated for psoriasis with methotrexate and etanercept over 10 years. The disease was poorly controlled with this regimen, and adalimumab therapy was initiated. Following 20 months of adalimumab therapy, the patient developed a myxofibrosarcoma at the site of the adalimumab injections (8). Another report described the development of a Merkel cell carcinoma in a patient with rheumatoid arthritis who received adalimumab, methotrexate and prednisone (9). A similar case report described a 61-year-old patient with rheumatoid arthritis who was treated with leflunomide and methylprednisolone between July 2005 and February 2008. Following initiation of adalimumab therapy, a lesion corresponding to Kaposi's sarcoma was detected in November 2008. Adalimumab was withdrawn in November 2009, and the patient was switched to low-dose corticosteroid treatment. By June 2010, complete resolution of the papulonodular lesions was observed (10). Another 67-year-old patient with rheumatoid arthritis developed Kaposi's sarcoma following treatment with adalimumab. The patient was initially treated with methotrexate and etanercept and achieved a 2-year remission. Following a psoriatic flare-up, etanercept was discontinued and adalimumab was added to the methotrexate regimen. Two months later, the patient developed skin lesions that were compatible with Kaposi's sarcoma (11).

The present case may be of interest to clinicians as, to the best of our knowledge, this is the first report of myxoid liposarcoma associated with adalimumab treatment. As mentioned above, several clinical reports have described some type of tumour associated with the use of biological agents; however, there is currently lack of evidence to support a possible association between the administration of anti-TNF- $\alpha$ agents and the risk of cancer (12).

Large-scale studies are warranted in the future to conclusively establish a direct (if any) association between tumour development and the administration of adalimumab.

\section{Acknowledgements}

The authors would like to thank Jose Carlos Medraño (Internal Medicine Department, Meixoeiro Hospital) for his assistance.

\section{Funding}

No funding was received.

\section{Availability of data and materials}

The datasets used and/or analysed during the present study are available from the corresponding author on reasonable request.

\section{Authors' contributions}

CRC, VMG and MCG followed up the patient, wrote the manuscript and designed the study. Moreover, they contributed to addressing all questions related to the accuracy and integrity of this study. JINV and CFV provided the histopathological results. All authors read and approved the final version of the manuscript.

\section{Ethics approval and consent to participate}

The authors declare that the procedures followed were in accordance with the ethical standards of the responsible committee on human experimentation (institutional and national) and with the Helsinki Declaration of 1975, as revised in 2000 (5).

\section{Patient consent for publication}

Written informed consent was obtained from the patient regarding the publication of the case details and any accompanying images.

\section{Competing interests}

The authors declare no potential competing interests with respect to the research, authorship and/or publication of this article.

\section{References}

1. Augustin M, Langenbruch A, Gutknecht M, Reich K, Körber A, Maaßen D, Mrowietz U, Thaçi D, von Kiedrowski R and Radtke MA: Definition of psoriasis severity in routine clinical care: Current guidelines fail to capture the complexity of long-term psoriasis management. Br J Dermatol 179: 1385-1391, 2018.

2. Carrera CG, Dapavo P, Malagoli P, Naldi L, Arancio L, Gaiani F, Egan CG, Di Mercurio M and Cattaneo A: PACE study: Real-life Psoriasis Area and Severity Index (PASI) 100 response with biological agents in moderate-severe psoriasis. J Dermatolog Treat 29: 481-486, 2018.

3. Billi AC and Gudjonsson JE: Adalimumab in Psoriasis: How much is enough? J Invest Dermatol 139: 19-22, 2019.

4. Esposito M, Prignano F, Rongioletti F, Hansel K, Bianchi L, Pescitelli L, Lazzeri L, Ricceri F, Mugheddu C, Bavetta M, et al: Efficacy and safety of adalimumab after failure of other anti-TNF $\alpha$ agents for plaque-type psoriasis: Clinician behavior in real life clinical practice. J Dermatolog Treat 6: 1-5, 2018.

5. Ruiz V, Velásquez M and Barrera LF: Immunogenetic aspects of psoriasis with emphasis on micro-RNA. Inmunol 33: 137-146, 2014.

6. Chiesa Fuxench ZC, Shin DB, Ogdie Beatty A and Gelfand JM: The risk of cancer in patients with psoriasis: A populationbased cohort study in the health improvement network. JAMA Dermatol 152: 282-290, 2016

7. Rønholt K and Iversen L: Old and new biological therapies for psoriasis. Int J Mol Sci 18: pii: E2297, 2017.

8. Farshid G, Prowse P and True B: Epithelioid myxofibrosarcoma developing at the injection site of Adalimumab therapy for psoriatic synovitis. Eur J Rheumatol 5: 131-134, 2018.

9. Krishna SM and Kim CN: Merkel cell carcinoma in a patient treated with adalimumab: Case report. Cutis 87: 81-84, 2011.

10. Amadu V, Satta R, Montesu MA and Cottoni F: Kaposi's sarcoma associated with treatment with adalimumab. Dermatol Ther (Heidelb) 25: 619-620, 2012.

11. Bret J, Hernandez J, Aquilina C, Zabraniecki L and Fournie B: Kaposi's disease in a patient on adalimumab for rheumatoid arthritis. Joint Bone Spine 76: 721-722, 2009.

12. Bonovas S, Minozzi S, Lytras T, González-Lorenzo M, Pecoraro V, Colombo S, Polloni I, Moja L, Cinquini M, Marino V, et al: Risk of malignancies using anti-TNF agents in rheumatoid arthritis, psoriatic arthritis, and ankylosing spondylitis: A systematic review and meta-analysis. Expert Opin Drug Saf 15 (Suppl 1): 35-54, 2016 